\title{
PRODUCCIÓN DE METANO EN REACTORES MONOFÁSICO Y BIFÁSICO UTILIZANDO AGUA MODELADA DE CAFÉ SOLUBLE
}

\author{
METHANE PRODUCTION IN SINGLE PHASE AND BIPHASE \\ REACTORS USING MODELED SOLUBLE COFFEE WATER
}

\author{
Mabel Leonela Laz Mero ${ }^{12}$, Miguel Alejandro Tuárez Párraga ${ }^{13}$, Dennys Lenin Zambrano Velásquez ${ }^{1}$, Rosa Alexandra \\ Córdova Mosquera ${ }^{2}$ \\ 'Posgrado Agroindustria. Escuela Superior Politécnica Agropecuaria Manabí. Sitio El limón, Calceta, Manabí - Ecuador \\ ${ }^{2}$ Universidad Técnica de Manabí, Av. Urbina y Ché Guevara. Portoviejo, Manabí - Ecuador \\ ${ }^{3}$ La Fabril S.A. Ubicación: Montecristi, Km 5 ำ2 vía Manta, Manabí - Ecuador \\ Email: mabel_laz@hotmail.com
}

\section{Información del artículo}

Tipo de artículo: Artículo original

\section{Recibido:}

18/02/2021

Aceptado:

$15 / 12 / 2021$

Licencia:

CC BY-NC-SA 4.0

Revista

ESPAMCIENCIA

12(2):84-94

DOI:

https://doi.org/10.5 1260/revista_espam ciencia.v12i 2.243

\begin{abstract}
Resumen
El objetivo de la presente investigación fue establecer la producción de metano en reactores con Sistema Monofásico (SM) y Sistema Bifásico (SB) utilizando como sustrato agua modelada de la industria de café soluble, con una demanda química de oxígeno (DQO) de 7,5 g/L, considerado como un residuo con alta carga orgánica. Las pruebas de laboratorio se realizaron en reactores provistos con filtro anaeróbicos de flujo ascendente (FAFA), evaluando parámetros de: pH, relación entre ácidos grasos volátiles y alcalinidad (FOSTAC), remoción DQO, concentración y rendimiento de metano, aplicando métodos normalizados para el análisis de aguas residuales descrita por APHA y WPCF, 2012. En la variable rendimiento el SM mostró valores promedios de $0,15 \mathrm{dm}^{3} \mathrm{CH}_{4} / \mathrm{gDQO}$ en comparación a los exhibidos por el SB con valores promedios de 0,26 $\mathrm{dm}^{3} \mathrm{CH}_{4} / \mathrm{g}$ DQO, los rangos de operación del SB fueron: $\mathrm{pH}(6,38-8,0)$, FOSTAC $(0,42-0,60)$, AGV(160-423 $\mathrm{mg} / \mathrm{l})$, remoción DQO (61-85\%); donde se comprobó estadísticamente que las producciones de metano en los sistemas evaluados difieren significativamente $(\mathrm{p}<0,05)$, evidenciando que en el SB se incrementó los valores promedios de producción de metano del $70 \%$ en relación al SM.
\end{abstract}

Palabras clave: Agua modelada, sistemas de reactores, rendimiento de metano

\begin{abstract}
The objective of this research was to establish the production of methane in reactors with a Single-Phase System (SM) and a Two-Phase System (SB) using modeled water from the soluble coffee industry as a substrate, with a chemical oxygen demand (COD) of 7, $5 \mathrm{~g} / \mathrm{L}$, considered as a residue with a high organic load. The laboratory tests were carried out in reactors equipped with an up flow anaerobic filter (FAFA), evaluating parameters of: $\mathrm{pH}$, relationship between volatile fatty acids and alkalinity (FOSTAC), COD removal, concentration and methane performance, applying standardized methods for the wastewater analysis described by APHA and WPCF, 2012. In the performance variable, the SM showed average values of $0.15 \mathrm{dm}^{3} \mathrm{CH}_{4} / \mathrm{gDQO}$ compared to those exhibited by the SB with average values of $0.26 \mathrm{dm}^{3} \mathrm{CH}_{4} / \mathrm{g} \mathrm{COD}$, the SB operating ranges were: $\mathrm{pH}(6.38-$ 8.0), FOSTAC (0.42-0.60), AGV (160-423 mg / 1), COD removal (61-85\%); where it was statistically verified that methane productions in the evaluated systems differ significantly $(\mathrm{p}<0.05)$, showing that in the BS the average methane production values increased by $70 \%$ in relation to the SM.
\end{abstract}

Keywords: Modeled water, reactor systems, methane performance. 


\section{INTRODUCCIÓN}

Actualmente, la bebida de café es un paradigma a nivel mundial, incrementando su consumo en países desarrollados (Murthy y Naidu, 2012); hasta el 50\% de su producción desde los años 1990 hasta el 2015. Por tal motivo, países como Brasil, Vietnam, Colombia, Indonesia y Etiopia lideran la producción mundial de este fruto, llegando a cosechar hasta 2594100 kilogramos en el año 2015 en Brasil (Lazcano, 2015).

Yánez (2018) manifiesta que la superficie del Ecuador para el cultivo de café abarca aproximadamente el 30\% de su territorio nacional, siendo así que, Ecuador es el cuarto exportador sudamericano más grande de café soluble a Rusia (El Telégrafo, 2017).

Por consiguiente, el café es el segundo producto más vendido en el mundo después del petróleo, por lo cual se genera gran cantidad de desperdicios durante su producción, a esto se suma que existe un tercio de la producción mundial de alimentos que se desperdician (Restrepo et al., 2011), constituyendo una fuente de contaminación hacia las reservas de agua, lo que representa el 9,6\% de las emisiones mundiales de $\mathrm{CO} 2$ (Fisgativa et al., 2017).

Producto de aquello, la industria cafetera utiliza grandes cantidades de agua durante su procesamiento, estimando de 40 a $45 \mathrm{~L}$ de vertido residual por kilogramo de café procesado (Blinová et al., 2017). Los efluentes contienen alta carga orgánica, obteniendo desechos o subproductos definidos mundialmente como un problema debido a la falta de conocimientos sobre su adecuado tratamiento (Vargas y Pérez, 2018); (Saval, 2012).

Por estas razones, durante los últimos años ha existido un creciente interés en el desarrollo y/o la optimización de tecnologías para el aprovechamiento de los residuos con alta carga orgánica generados por las empresas agroindustriales procesadoras de café, en países industrializados como Brasil y en vías de desarrollo como Colombia.

Inclusive, se oferta una alternativa tecnológica atractiva como la DA, en la cual se obtiene producto como el metano con capacidad energética y lodos con propiedades fertilizantes (Mussatto et al., 2011). Podemos recalcar lo mencionado por Ramírez (2012); Peñaranda et al., (2017) quienes afirman que el aprovechamiento de estos residuos es una alternativa que impulsa el desarrollo de tecnologías, orientadas hacia una transformación sustentable de los recursos naturales (Rosas et al., 2016).

Por tal motivo, la digestión anaerobia ofrece una oportunidad viable de aprovechamiento de estos residuos orgánicos mediante un proceso de degradación bioquímica en ausencia de oxígeno (González et al., 2015). Según FAO (2013) y González (2009) argumentan que la bioenergía se obtiene a base de desechos orgánicos agroindustriales; siendo esta, una manera de aprovecharlos mediante procesos biotecnológicos (Chen et al., 2008); proponiendo de esta manera aportes y pertinencias al sector agroindustrial.

El presente estudio tiene como objetivo establecer la producción de metano en reactores con Sistema Monofásico (SM) y Sistema Bifásico (SB) para posteriormente compararlos entre sí; por consiguiente, se realizaron los respectivos análisis de $\mathrm{pH}$, FOSTAC, AGV, remoción DQO, rendimiento y concentración de metano.

\section{MATERIALES Y MÉTODOS}

La investigación se desarrolló en el laboratorio de Operaciones Unitarias y en el Laboratorio de Análisis Químico y Biotecnológico de la Universidad Técnica de Manabí. Para la aplicación de la metodología utilizada en la investigación se tomó como punto de partida: la modelación y caracterización del agua residual de la industria de café instantáneo, preparación del inóculo, las operaciones de carga, arranque de los reactores, métodos analíticos y análisis estadístico con pruebas paramétricas (ADEVA) y no paramétricas (U de Mann-Whitney), mediante el programa estadístico SPSS.

\section{Agua residual modelada}

Se modeló agua residual de la industria de café soluble con características similares a la industria. Cabe mencionar que el agua residual se recolectó de una de las industrias de café instantáneo del país, ubicada en la provincia de Manabí. El sustrato inicialmente es una solución concentrada, mediante la adición de $20 \mathrm{~g}$ de café tostado y molido (Fernández y Foster 1993), en 1 L de agua destilada sometida a calentamiento hasta ebullición, posteriormente se filtra la solución mediante gasa fina.

Se definió el volumen de agua destilada de acuerdo a la ecuación 1 propuesta por Morales y Vera (2018), para modelar el efecto de dilución en la muestra concentrada a partir de un balance de materia y fijar la carga orgánica expresada como DQO en $7500 \mathrm{mg} / \mathrm{L}$.

$$
V_{H 2 O}=\frac{V_{o}-D Q O_{O}}{D Q O_{F}}-V_{O}
$$

Donde:

$V_{H 2 O}$ : Volumen de agua añadida, $\mathrm{ml}$

$V_{o}$ : Volumen de agua concentrada, $\mathrm{ml}$

$D Q O_{o}$ : Demanda química de oxígeno inicial, $\mathrm{mg} / \mathrm{L}$

$D Q O_{F}$ : Demanda química de oxígeno final, $\mathrm{mg} / \mathrm{L}$ 


\section{Preparación del inóculo}

El inóculo se recolectó de un reactor anaerobio del tratamiento de residuos de la industria atunera, se almacenó en un envase de 20 L herméticamente sellado y transportado a las instalaciones del laboratorio. Se realizó proceso de desgasificación para evitar el ingreso de oxígeno al sistema durante 30 días, se aclimató alimentando semanalmente con solución de agua residual modelada con una concentración del $25 \%(\mathrm{p} / \mathrm{v})$ en sacarosa.

Para su caracterización se tomó una muestra representativa para realizar sólidos suspendido volátiles (SSV); según la norma alemana VDI 4630 (2006) la cual indica que la cantidad de sólidos volátiles debe ser $\geq 50 \%$ para ser inoculado en los reactores, caso contrario se continúa alimentando hasta alcanzar dicho valor.

\section{Descripción de reactores}

Los reactores de los SM y SB son de tipo FAFA, está construido de acero inoxidable de $2 \mathrm{~mm}$ de espesor. Poseen una altura de $35 \mathrm{~cm}$ y un diámetro de $14 \mathrm{~cm}$, a su vez está recubierto de un cilindro de $23 \mathrm{~cm}$ de diámetro y $24,5 \mathrm{~cm}$ de alto; este último actúa como un sistema de calentamiento mediante una resistencia; como medio de empaque se utilizó tubería corrugada $\mathrm{PVC}$ de $2,5 \mathrm{~cm}$ de alto, $15 \mathrm{~mm}$ de diámetro exterior, $11 \mathrm{~mm}$ de diámetro interior y $0,55 \mathrm{~g}$ de peso. El volumen de reacción calculado fue de $3,956 \mathrm{~L}$.

En el SM se desarrolló las cuatro fases del proceso de DA simultáneamente en un único reactor (Hid, Aci, Ace y Met), como se muestra en la figura 1.

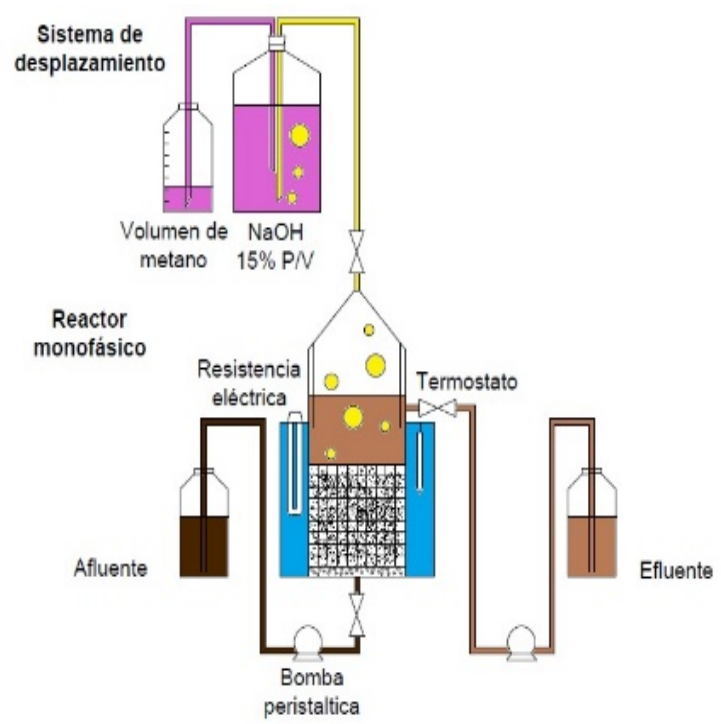

Figura 1. Reactor sistema monofásico

Mientras que en el SB se separan las fases de la siguiente manera: proceso en el primer reactor (Hid-Aci), proceso en el segundo reactor (Ace-Met), como se muestra en la figura 2.

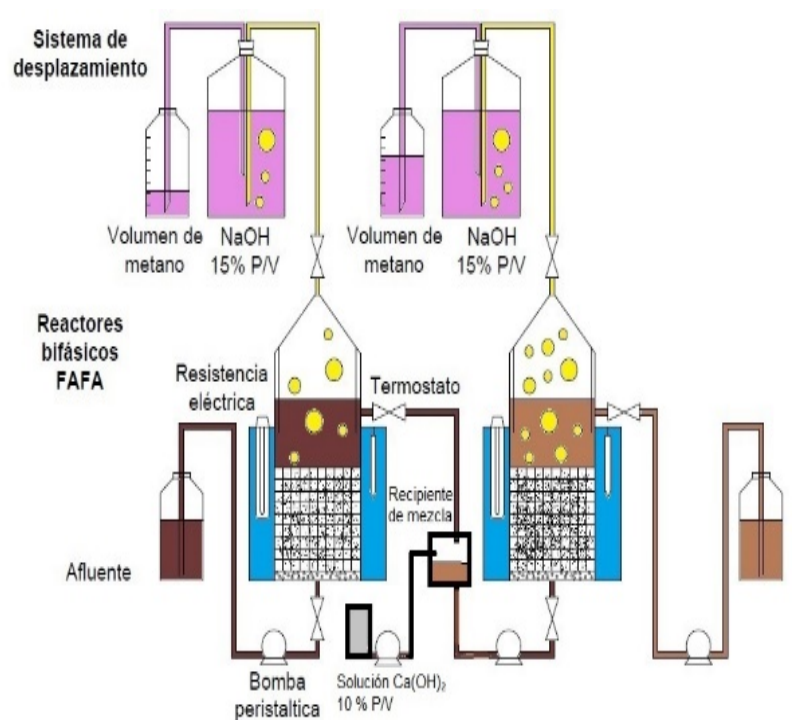

Figura 2. Reactores sistemas bifásicos

\section{Arranque del SM}

Para el arranque del reactor se formuló una mezcla del $40 \%$ inóculo y $60 \%$ agua modelada en relación al volumen de reacción, sellando herméticamente el reactor, se estableció la temperatura de $35^{\circ} \mathrm{C}$ y se recirculó la mezcla mediante una bomba peristáltica Jebao DP-4 durante un periodo de 15 días para asegurar que la biomasa activa se adhiera al empaque.

Durante 7 días se alimentó de manera semicontinuo cada $24 \mathrm{~h}$ un caudal de $105,5 \mathrm{ml} / \mathrm{d}$ correspondiente a una carga orgánica volumétrica inicial (Bv) de 0,2 g L-1. d-1, culminado los 15 días se aumentó la Bv, de acuerdo a la estabilidad del proceso de DA. Adicionalmente el sistema de desplazamiento Boyle-Mariotte se conectó a la campana del reactor mediante una manguera de $1 / 4$ in donde circula biogás y entra en contacto directo con la solución del $\mathrm{NaOH}$ al 15\% P/V.

\section{Arranque de los reactores en el SB}

Para mayor apreciación se llamó al reactor A donde se desarrolló la Hid-Aci y al reactor B donde se desarrolló la Ace-Met. Ambos reactores A y B son inoculados mediante la mezcla $40 \%$ inóculo y $60 \%$ agua modelada en relación al volumen de reacción. El reactor A se sometió a un tratamiento térmico (choque térmico) que consistió en aumentar la temperatura a $100 \pm 2^{\circ} \mathrm{C}$ durante un periodo de $2 \mathrm{~h}$ (Valdez y Poggi, 2009); para contribuir al enriquecimiento de bacterias celulolíticas formadoras de esporas; posteriormente se estableció la temperatura de $35^{\circ} \mathrm{C}$ y se recirculó la mezcla mediante una bomba peristáltica Jebao DP-4 durante un periodo de 15 días 
para asegurar que la biomasa activa se adhiera el empaque.

Transcurrido 7 días se alimentó de manera semicontinua cada 24 h un caudal de 105,5 mL.d-1 correspondiente a una carga orgánica volumétrica inicial (Bv) de 0,2 g L-1 d-1, el efluente del reactor $\mathrm{A}$ alimenta al reactor $\mathrm{B}$; culminado los 15 días se aumentó la Bv con base en la estabilidad que brinde el proceso en cuanto a sus variables de control. Ambos reactores poseen su propio sistema de desplazamiento Boyle-Mariotte, bajo el mismo principio de desplazamiento descrito en el SM.

\section{Cálculo del caudal volumétrico (Qv) y el tiempo de retención hidráulica (TRH)}

Una vez estabilizados los reactores y el inóculo aclimatado, se incrementó periódicamente la carga orgánica volumétrica $(\mathrm{Bv})$, en función de los resultados observados de las variables de control, para determinar el rendimiento de metano. Para establecer el caudal volumétrico y tiempo de retención hidráulico se utilizó las Ecuaciones 2; 3 y 4 propuestas por Diaz (2018).

Donde:

$$
Q_{m}=B_{v} * V_{r}
$$

$Q_{m}:$ Caudal másico, $\mathrm{g} \mathrm{d}^{-1}$

$B_{v}$ : Carga orgánica volumétrico, $\mathrm{g} \mathrm{L}^{-1} \mathrm{~d}^{-1}$.

$V_{r}$ : Volumen de reacción, $\mathrm{L}$

El caudal volumétrico se define por la siguiente expresión:

$$
Q_{v}=\frac{Q_{m}}{C O_{D Q O}}
$$

Donde:

$Q_{m}$ : Caudal másico, $\mathrm{g} \mathrm{d}^{-1}$

$Q_{v}$ : Caudal volumétrico, $\mathrm{L} \mathrm{d}^{-1}$

$C O_{D Q O}$ : Carga orgánica, $\mathrm{g} \mathrm{L}^{-1}$

El tiempo de retención hidráulica (TRH) se define como:

Donde:

$$
T R H=\frac{V_{r}}{Q_{v}}
$$

THR: Tiempo de retención hidráulica, $\mathrm{d}$

$Q_{v}$ : Caudal volumétrico, $\mathrm{L} \mathrm{d}^{-1}$

$V_{r}$ : Volumen de reacción, $\mathrm{L}$

\section{Método de evaluación}

Los ensayos analíticos empleados en la investigación se realizaron según los métodos normalizados para el análisis de aguas residuales (APHA y WPCF, 2012) especificados en el cuadro 1.
Cuadro 1. Métodos aplicados según las variables en estudio

\begin{tabular}{ccc}
\hline Parámetro & Método & Equipo \\
\hline $\begin{array}{c}\text { Potencial de } \\
\text { hidrógeno }\end{array}$ & Potenciométrico & Fisher Scientific Accumet \\
DQO & Espectrofotómetro & Thermo Scientific - Evolution \\
UV-Vis & 60 S \\
DBO $_{5}$ & Respirométrico & DBO hach - bod trak II \\
ST, SF y SV & Gravimétrico & Memmert D-91126 \\
Relación AGV/Alc & Potenciométrico/ & Fisher Scientific Accumet \\
valoración & AB150 \\
bomposición del & Medidor de gases & Multitec ${ }^{\circledR} 545$ \\
\hline
\end{tabular}

\section{RESULTADOS Y DISCUSIÓN}

Se evaluaron los resultados obtenidos durante la producción de metano en los SM y SB, utilizando agua modelada de la industria de café soluble, iniciando con la caracterización del agua residual y del inóculo, los cuales son las entradas fundamentales para implementar el arranque y posterior seguimiento de una forma continua a los reactores durante 13 semanas, validando el comportamiento de parámetros fisicoquímicos tales como: pH, FOSTAC, AGV, remoción DQO, rendimiento y concentración de metano, comparando los sistemas con pruebas paramétricas y no paramétricas, mediante el programa estadístico SPSS.

\section{Caracterización del agua residual.}

En la presente investigación se modeló agua residual de la industria de café soluble, con base en lo sugerido por García et al. (1995) y por Fernández y Forster (1993) quienes evalúan un extracto muy similar al proceso de producción de café instantáneo, con lo cual se obtuvo los siguientes resultados que se muestran en el cuadro 2 .

En la caracterización del agua modelada se puede evidenciar un $\mathrm{pH}$ ácido 5,4, un DQO de $7500 \mathrm{mg} / \mathrm{L}$ y un índice de biodegradabilidad 0,55 producto de la relación entre DBO5/DQO, lo que indica que es un sustrato con capacidad para degradación biológica, de acuerdo a lo reportado por Crites y Tchobanoglous (2000).

Cuadro 2. Métodos aplicados según las variables en estudio

\begin{tabular}{ccc}
\hline Parámetro & $\begin{array}{c}\text { Agua residual de la } \\
\text { industria }\end{array}$ & $\begin{array}{c}\text { Agua residual } \\
\text { modelada }\end{array}$ \\
\hline $\mathrm{pH}$ & $5,25 \pm 1,25$ & $5,40 \pm 0,8$ \\
$\mathrm{DBO}_{5}(\mathrm{mg} / \mathrm{L})$ & $3397,8 \pm 1090$ & $4129,2 \pm 118$ \\
$\mathrm{DQO}(\mathrm{mg} / \mathrm{L})$ & $6124,8 \pm 2608$ & $7496,73 \pm 80$ \\
$\begin{array}{c}\text { Índice de } \\
\text { biodegradabilidad }\end{array}$ & $0,5 \pm 0,09$ & $0,55 \pm 0,03$ \\
\hline $\mathrm{n}=3$ \\
Promedio \pm desviación estándar
\end{tabular}




\section{Caracterización del inóculo}

Durante el período de adaptación de los reactores se caracterizó el inóculo obteniendo los valores reportados en el cuadro 3. De acuerdo a lo establecido por la VDI 4630 (2006) este inóculo cumple con los requisitos para el adecuado inicio de la DA, debido a que su contenido de los SV en base seca fue mayor al $50 \%$.

Cuadro 3. Caracterización del inóculo

\begin{tabular}{ccc}
\hline \multicolumn{1}{c}{ Parámetro } & Unidad & Valores \\
\hline sólidos totales (ST) & $\mathrm{gST} / \mathrm{L}$ & $0,0502 \pm 0,0032$ \\
volátiles (SV) & $\mathrm{gSV} / \mathrm{L}$ & $0,0309 \pm 0,0035$ \\
suspendidos volátiles (SSV) & $\mathrm{gSSV} / \mathrm{L}$ & $0,0230 \pm 0,0033$ \\
disueltos volátiles (SDV) & $\mathrm{gSDV} / \mathrm{L}$ & $0,0079 \pm 0,0025$ \\
\hline $\mathrm{n}=3$ & & \\
Promedio \pm desviación estándar & &
\end{tabular}

\section{Evaluación de variables con seguimiento diario}

Como se evidencia en el cuadro 4, según prueba de normalidad de Kolmogorov - Smirnov, las variables de seguimiento diario (rendimiento de metano, $\mathrm{pH}, \mathrm{AGV}$ y FOSTAC) no siguen una distribución normal, por tanto, se realiza una prueba no paramétrica de U de MannWhitney.

Cuadro 4. Pruebas de normalidad para variables con seguimiento diario

\begin{tabular}{llll}
\hline & \multicolumn{2}{l}{ Kolmogorov } & -Smirnov \\
& Estadístico & gl & Sig. \\
\hline Rendimiento de Metano & 1,882 & 180 & 0,002 \\
pH & 1,737 & 180 & 0,005 \\
AGV & 1,818 & 180 & 0,003 \\
FOSTAC & 1,956 & 180 & 0,001 \\
\hline
\end{tabular}

No son significativamente diferentes $(\mathrm{p}>0.05)$

En el cuadro 5, de resumen de los ADEVA explican que uno de los sistemas (SM o SB) provocan cambios altamente significativos en las variables respuestas estudiadas.

Cuadro 5. Pruebas de U de Mann-Whitney de muestras independientes para variables con seguimiento diario

\begin{tabular}{|c|c|c|}
\hline \multicolumn{3}{|c|}{ Resumen de prueba de hipótesis } \\
\hline & Hipótesis nula & Sig. \\
\hline 1 & $\begin{array}{l}\text { La distribución de } \mathrm{dm} 3 \mathrm{CH} 4 / \mathrm{gDQO} \text { es la misma entre } \\
\text { las categorías de sistema. }\end{array}$ &, 000 \\
\hline 2 & $\begin{array}{l}\text { La distribución de } \mathrm{pH} \text { es la misma entre las categorías } \\
\text { de sistema. }\end{array}$ &, 000 \\
\hline 3 & $\begin{array}{c}\text { La distribución de } \mathrm{mg} / \mathrm{L} \text { es la misma entre las } \\
\text { categorías de sistema. }\end{array}$ & 000 \\
\hline 4 & $\begin{array}{c}\text { La distribución de FOSTAC es la misma entre las } \\
\text { categorías de sistema. }\end{array}$ & ,000 \\
\hline
\end{tabular}

\section{Rendimiento de metano}

Como se aprecia en el gráfico 1 , se representan los sistemas evaluados en el eje " $\mathrm{X}$ " y al rendimiento de metano en el eje "Y", el cual es expresado en $\mathrm{dm}^{3}$ $\mathrm{CH}_{4} / \mathrm{gDQO}$, los resultados obtenidos mediante la prueba de $\mathrm{U}$ de Mann-Whitney para el rendimiento de $\mathrm{CH}_{4}$ son menor a 0,05 , es decir se rechaza la hipótesis nula y se acepta la hipótesis alternativa, con lo que se determina estadísticamente diferencias significativas entre ambos sistemas. Lo que concuerda con Chinchilla (2013) reportando que valores de producción de metano en el $\mathrm{SB}$ es mayor al SM, debido a que el $\mathrm{SB}$ reúne las condiciones óptimas para la DA.

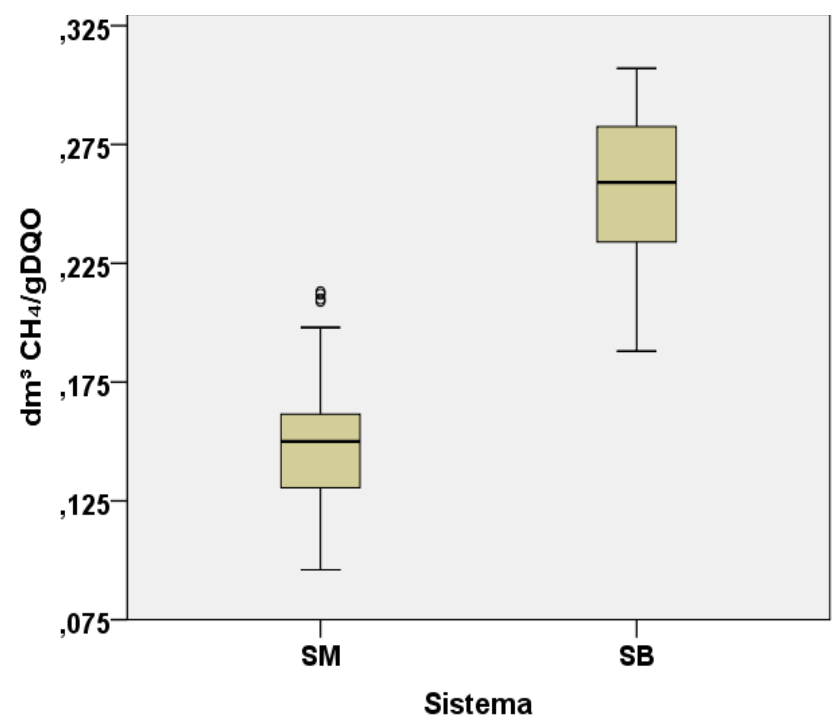

Gráfico 1. Comparación de gráficas de caja para rendimiento de metano entre los SM y SB

Existe datos atípicos que se evidencian en el gráfico 1, por lo que se procede a realizar la prueba de Moses para constatar la significancia de dichos valores, en el cuadro 6 se evidencia estadísticamente que los valores atípicos del SM son altamente significativos como se muestra en el gráfico 2 .

Cuadro 6. Pruebas de Moses de reacción extrema de muestras independientes para rendimiento de $\mathrm{CH}_{4}$

\begin{tabular}{|lll}
\hline & \multicolumn{2}{c}{$\begin{array}{c}\text { Resumen de prueba de hipótesis } \\
\text { Hipótesis nula }\end{array}$} \\
\hline $\mathbf{1}$ & $\begin{array}{l}\text { El intervalo de Rendimiento. } \mathrm{CH}_{4} \text { es el } \\
\text { mismo entre las categorías de sistema. }\end{array}$ & $1,207 \mathrm{E}-34^{1}$ \\
\hline & 1Se muestra la significancia exacta para esta prueba. \\
\hline
\end{tabular}




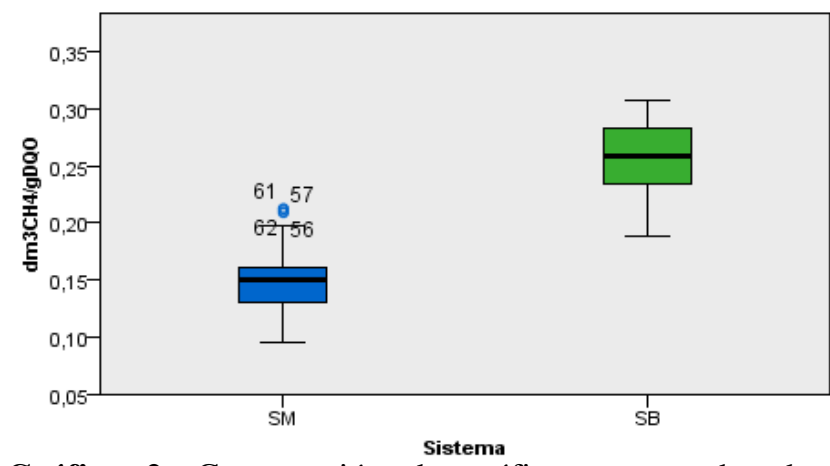

Gráfico 2. Comparación de gráficas en prueba de Mosses para rendimiento de metano entre los SM y SB

\section{pH}

Como se aprecia en el gráfico 3, los valores de $\mathrm{pH}$ obtenidos mediante la prueba de U de Mann-Whitney para el $\mathrm{pH}$ presentaron diferencias significativas $(\mathrm{p} \geq 0,05)$.

El SB presentó mayor estabilidad de los resultados de $\mathrm{pH}$ en comparación a los del SM, debido a que durante la separación de fases del SB en el reactor acidogénico los compuestos orgánicos solubles son convertidos en ácidos orgánicos lo que facilita la DA en la siguiente etapa del reactor metanogénico, por lo cual es más fácil controlar un $\mathrm{pH}$ en donde la mayoría de su población de microorganismos se encuentra en ambiente neutroalcalino, lo que concuerda con Göblös et al. (2008).

De similar manera Quintero y Rondón (2012) señalan que es muy importante mantener los valores de $\mathrm{pH}$ estable porque los cambios en este parámetro pueden afectar la población metanogénica que están presente en los lodos. Por tanto, Aristizábal (2015) plantea que se debe conocer la cantidad aproximada de base $(\mathrm{NaOH})$ que debe ser agregada para neutralizar el sustrato. Adicionalmente, existen datos atípicos que se evidencian en el gráfico 3 , por lo que se procede a realizar la prueba de Moses para contrastar la significancia de dichos valores mostrado en el gráfico 4.

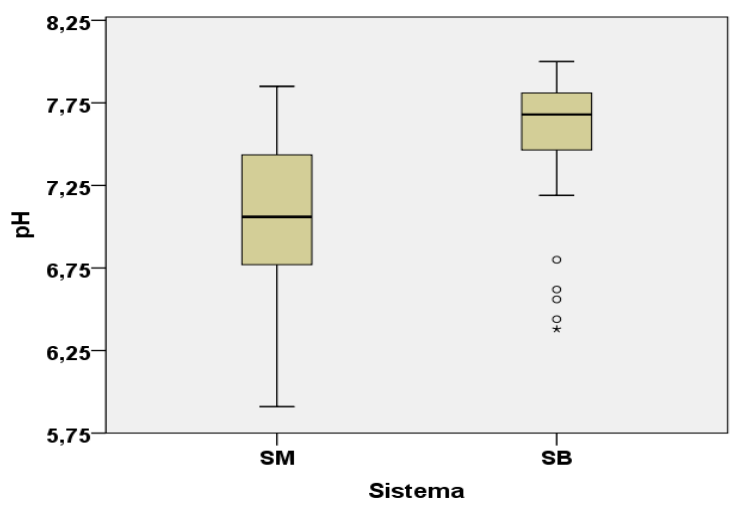

Gráfico 3. Comparación de gráficas de caja para $\mathrm{pH}$ entre los SM y SB.
De acuerdo a la prueba de Moses del cuadro 7, los valores atípicos del sistema $\mathrm{SB}$ son altamente significativos. Los cuales son evidenciados en el gráfico 4.

Cuadro 7. Pruebas de Moses de reacción extrema de muestras independientes para $\mathrm{pH}$

\begin{tabular}{|c|c|c|}
\hline \multicolumn{3}{|c|}{ Resumen de prueba de hipótesis } \\
\hline & Hipótesis nula & Sig. \\
\hline 1 & $\begin{array}{c}\text { El intervalo de pH es el mismo entre las } \\
\text { categorías de Sistema. }\end{array}$ & $6,632 \mathrm{E}-4^{1}$ \\
\hline \multicolumn{3}{|c|}{${ }^{1}$ Se muestra la significancia exacta para esta prueba. } \\
\hline
\end{tabular}

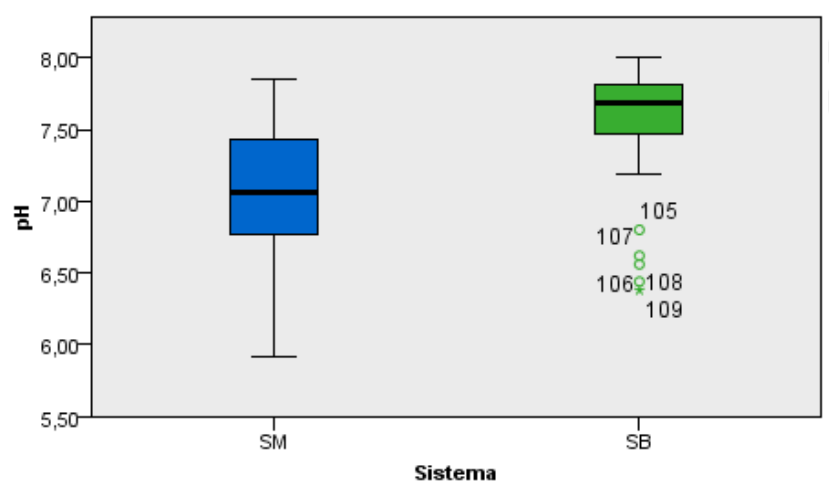

Gráfico 4. Comparación de gráficas en prueba de Moses para $\mathrm{pH}$ entre los SM y SB

\section{AGV}

Como se aprecia en el gráfico 5, se representan los sistemas evaluados en el eje " $\mathrm{X}$ " y los valores de AGV en el eje "Y", los resultados obtenidos mediante la prueba de U de Mann-Whitney son menor a 0,05 ; es decir se rechaza la hipótesis nula y se acepta la hipótesis alternativa, con lo que se determina estadísticamente diferencias significativas entre ambos SD, evidenciando el SB produce ligeramente mayores niveles de ácidos grasos volátiles $(\mathrm{mg} / \mathrm{L})$ con mayor estabilidad que en el SM, lo que concuerda con lo reportado por Ashley y Hurst (1981); Speece y Parking (1983) que establecen que la concentración de AGV no debe exceder de los 500 $\mathrm{mg} / \mathrm{L}$ para el desarrollo óptimo del proceso metanogénico. De similar manera los valores mínimos del SB de la presente investigación son análogos a los reportados por Guardia (2012) en su estudio de DA en SB. Por otra parte, Bolte, Hill, y Wood (1986); Braun, Huber y Meyrath (1981) realizaron estudios con desechos de cerdos donde reportan valores menores de 10 y hasta $527 \mathrm{mg} / \mathrm{L}$. 


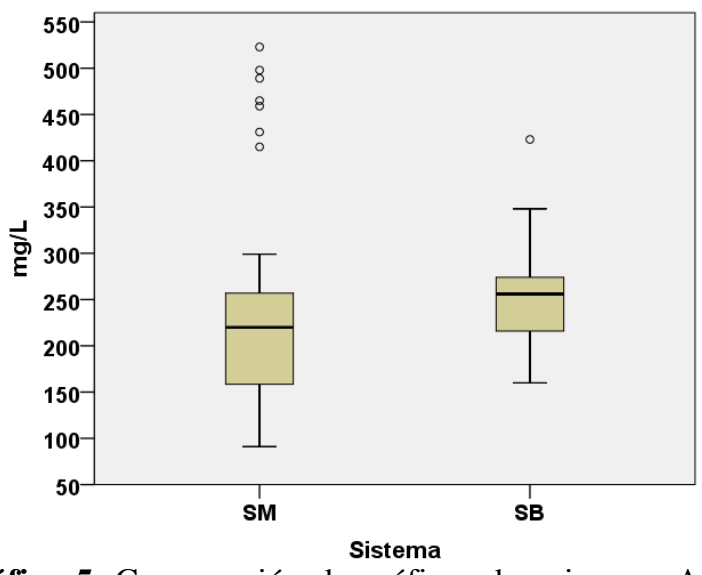

Gráfico 5. Comparación de gráficas de caja para AGV entre los SM y SB

De acuerdo a la prueba de Moses del cuadro 8, los valores atípicos del sistema no son significativos, los cuales se pueden visualizar en el gráfico 6 .

Cuadro 8. Pruebas de Moses de reacción extrema de muestras independientes para AGV

\begin{tabular}{|c|c|c|}
\hline \multicolumn{3}{|c|}{ Resumen de prueba de hipótesis } \\
\hline 1 & $\begin{array}{l}\text { El intervalo de AGV es el mismo entre las } \\
\text { categorías de sistema. }\end{array}$ & $1,000^{1}$ \\
\hline & 1Se muestra la significancia exacta para est & ueba. \\
\hline
\end{tabular}

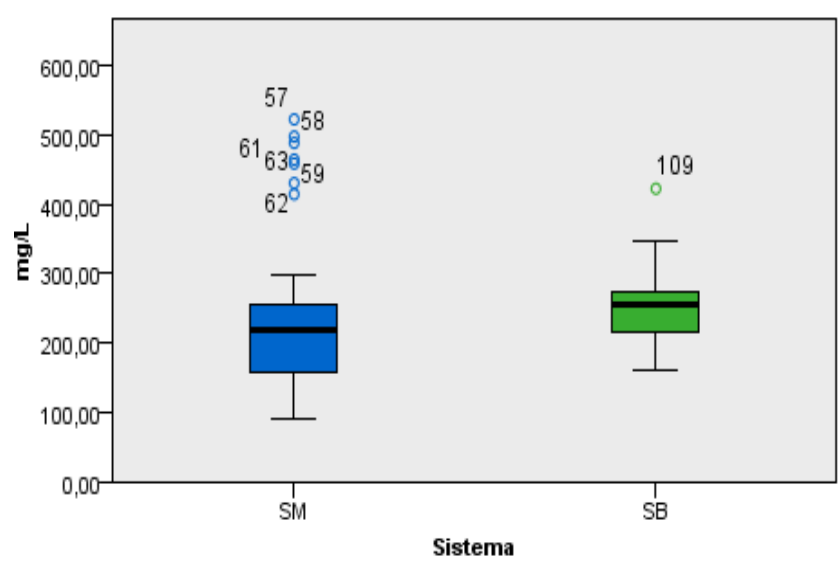

Gráfico 6. Comparación de gráficas en prueba de $\mathrm{Moses}^{\mathrm{o}}$ para AGV entre los SM y SB.

\section{FOSTAC}

Como se aprecia en la gráfico 7 , se representan los sistemas evaluados en el eje " $\mathrm{X}$ " y los valores de FOSTAC en el eje "Y", los resultados obtenidos mediante la prueba de U de Mann-Whitney son menor a 0,05 ; es decir se rechaza la hipótesis nula y se acepta la hipótesis alternativa, con lo que se determina estadísticamente diferencias significativas entre ambos
$\mathrm{SD}$, sin embargo los mejores valores de la relación de FOSTAC fueron efectuadas en el SB situándose entre 0,42 a 0,60 lo que concuerda con Lossie y Pütz (2011); Failla y Restuccia (2014) en donde mencionan que los rangos de FOSTAC es considerado estable cuando se encuentra entre 0,4 a 0,6 debido a que permite detectar a tiempo los problemas del proceso, hasta el inminente vuelco de la fase biológica del digestor, con lo que pueden tomarse contramedidas inmediatamente.

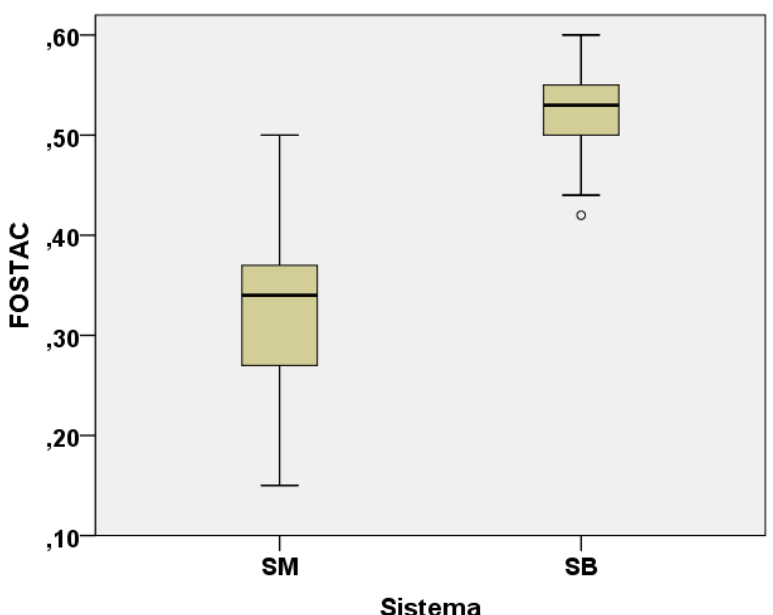

Gráfico 7. Comparación de pruebas U de Mann-Whitney para FOSTAC entre los SM y SB.

De acuerdo a la prueba de Moses del cuadro 9, los valores atípicos del sistema no son significativos, lo cual se aprecia en el gráfico 8 .

Cuadro 9. Pruebas de Moses de reacción extrema de muestras independientes para FOSTAC

\begin{tabular}{|c|c|c|}
\hline \multicolumn{3}{|c|}{ Resumen de prueba de hipótesis } \\
\hline 1 & $\begin{array}{l}\text { El intervalo de FOSTAC es el mismo entre las } \\
\text { categorías de sistema. }\end{array}$ & $1,604 \mathrm{E}-331$ \\
\hline \multicolumn{3}{|c|}{ ${ }^{1}$ Se muestra la significancia exacta para esta prueba. } \\
\hline
\end{tabular}

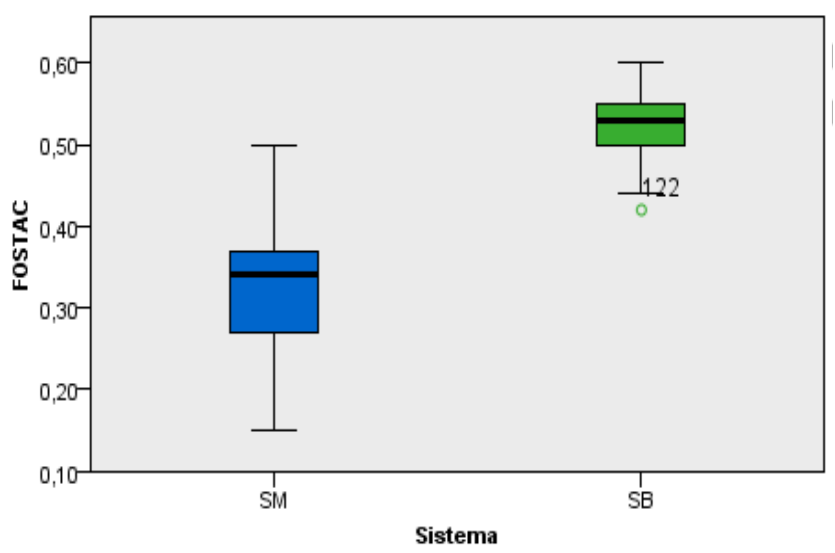

Gráfico 8. Comparación de gráficas en prueba de Moses para FOSTAC entre los SM y SB 


\section{Evaluación de variables con seguimiento semanal}

Como se evidencia en el cuadro 10, según la prueba de normalidad de Shapiro Wills, la remoción de DQO únicamente sigue una distribución normal en el SM a diferencia que en concentración de metano se presenta una distribución normal en ambos SD.

Cuadro 10. Pruebas de normalidad para variables con seguimiento semanal

\begin{tabular}{ccccc}
\hline & \multicolumn{4}{c}{ Shapiro-Wilk } \\
& Sistema & Estadístico & Gl & Sig. \\
\hline \multirow{2}{*}{ Remoción DQO } & SM & 0,936 & 13 & 0,406 \\
& SB & 0,851 & 13 & 0,029 \\
\multirow{2}{*}{ Concentración de metano } & SM & 0,960 & 13 & 0,756 \\
& SB & 0,870 & 13 & 0,053 \\
\hline
\end{tabular}

*. Este es un límite inferior de la significación verdadera.

a. Corrección de la significación de Lilliefors

No son significativamente diferentes $(\mathrm{p}>0,05)$

Como se evidencia en el cuadro 11, según la prueba de homogeneidad de varianza, resultó significativa para la remoción de DQO, a diferencia de la concentración de metano que no presentó diferencia significativa.

Cuadro 11. Prueba de homogeneidad de varianza para variables con seguimiento semanal

\begin{tabular}{ccccc}
\hline & & \multicolumn{3}{c}{ Levene } \\
& Estadístico & gl1 & gl2 & Sig. \\
\hline Remoción DQO & 7,658 & 1 & 24 & 0,011 \\
Concentración de metano & 0,829 & 1 & 24 & 0,371 \\
\hline
\end{tabular}

No son significativamente diferentes ( $p>0,05)$

Nota. Fuente: Autores de la investigación

La remoción de DQO no cumple con los supuestos de normalidad y homogeneidad de varianza por lo cual se utiliza una prueba no paramétrica, la prueba U de MannWhitney, en cambio la concentración de metano cumple con ambos supuestos, por tal antecedente se utiliza una prueba paramétrica de ADEVA.

En el cuadro 12 de resumen de los ADEVA se detalla que uno de los sistemas (SM o SB) provocan cambios altamente significativos en la remoción de DQO

Cuadro 12. Prueba de U de Mann-Whitney de muestras independientes para remoción de DQO

\begin{tabular}{|c|c|c|}
\hline \multicolumn{3}{|c|}{ Resumen de prueba de hipótesis } \\
\hline 1 & $\begin{array}{l}\text { La distribución de Remoción.DQO es la misma entre las } \\
\text { categorías de Sistema. }\end{array}$ &, $002^{1}$ \\
\hline & 1Se muestra la significancia exacta para esta prueba. & \\
\hline
\end{tabular}

\section{Remoción de DQO}

Como se aprecia en el gráfico 9, se representan los sistemas evaluados en el eje " $\mathrm{X}$ " y los valores de remoción de DQO en el eje "Y", los resultados obtenidos mediante la prueba de U de Mann-Whitney son menor a 0,05; es decir se rechaza la hipótesis nula y se acepta la minúscula

hipótesis alternativa, con lo que se determina estadísticamente diferencias significativas entre ambos $\mathrm{SD}$, por lo tanto, se evidencia que existe mayor remoción de DQO en el SB lo que concuerda con Guardia (2012) resaltando que un SB es más eficiente que un SM.

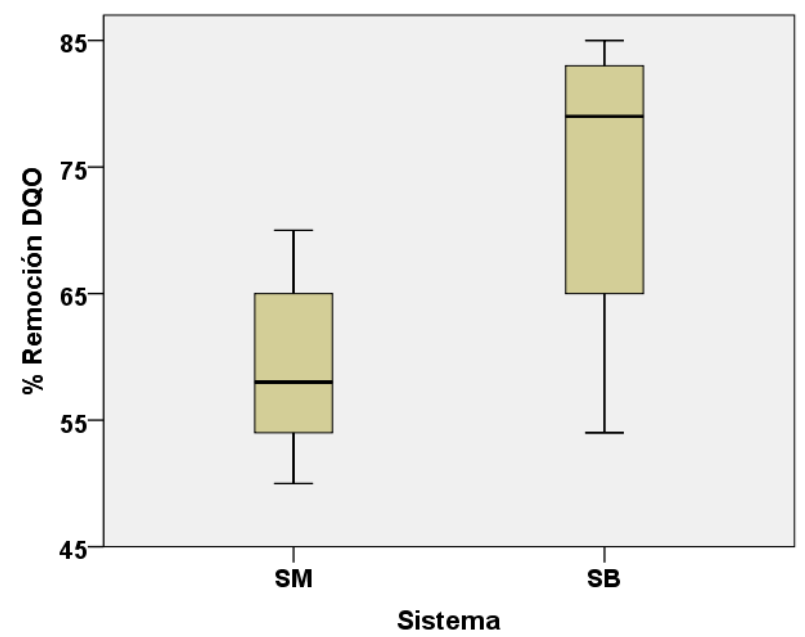

Gráfico 9. Comparación de gráficas de caja para remoción de DQO entre los SM y SB

En el cuadro 13, de resumen de ADEVA se evidencia que uno de los sistemas (SM o SB) provocan cambios altamente significativos en la concentración de metano.

Cuadro 13. Prueba de ADEVA para concentración de metano.

\begin{tabular}{lccccc}
\hline $\begin{array}{c}\text { Fuente de } \\
\text { Variación }\end{array}$ & $\begin{array}{c}\text { Suma de } \\
\text { cuadrados }\end{array}$ & Gl & $\begin{array}{c}\text { Redia } \\
\text { cuadrática }\end{array}$ & F & Sig. \\
\hline Tratamientos & 193,885 & 1 & 193,885 & 16,50 & 0,0 \\
Error & 282,000 & 24 & 11,750 & & \\
Total & 475,885 & 25 & & & \\
\hline Se muestran las significancias asintóticas. El nivel de significancia es \\
0,05
\end{tabular}

\section{Concentración de metano}

Como se aprecia en la gráfico 10 , se representan los sistemas evaluados en el eje " $\mathrm{X}$ " y los valores de concentración de metano en el eje "Y", los resultados obtenidos mediante la prueba de ADEVA son menor a 0,05 ; es decir se rechaza la hipótesis nula y se acepta la hipótesis alternativa, con lo que se determina estadísticamente diferencias significativas entre ambos $\mathrm{SD}$, evidenciando que el SM produce ligeramente mayor concentraciones de metano que en el SB, sin embargo, el rendimiento de metano es superior en el SB y los resultados concuerdan con valores similares reportados por Dinsdale et al. (1997); Scano et al. (2014) entre 45 a $60 \%$, siendo así que ambos sistemas son considerados 
combustibles según Varnero (2011), el cual indica que valores por encima de $45 \%$ de concentración de metano son inflamables.

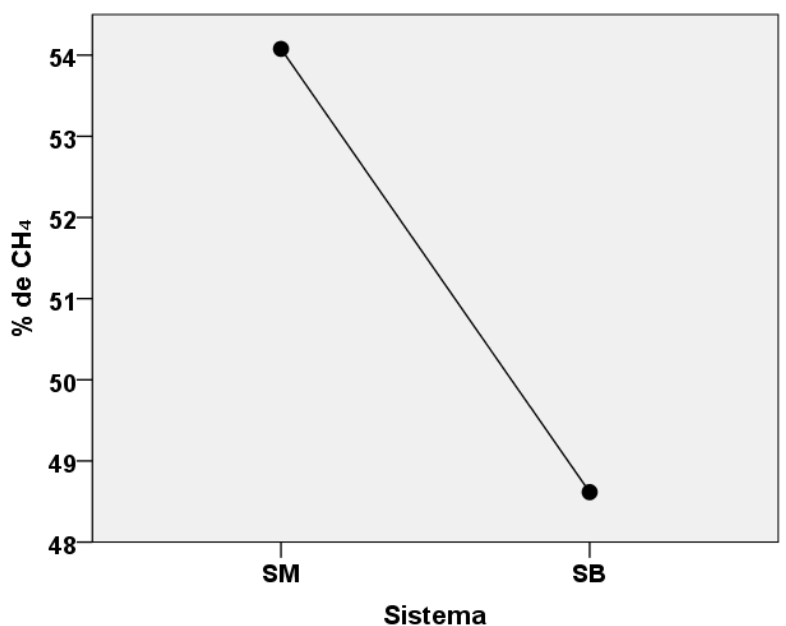

Gráfico 10. Comparación de medidas para concentración de metano entre los SM y SB

Adicionalmente, Guardia et al. (2014) en su estudio relacionado con digestión anaerobia de aguas residuales de café, obtuvo $61 \%$ de metano, encontrando concentraciones por encima de la presente investigación.

\section{CONCLUSIONES}

Se logró modelar las principales características fisicoquímicas del agua residual de la industrial de café instantáneo con una similitud del $86 \%$ con respecto al agua residual de este tipo de industria de café soluble.

El SB tuvo un incremento de producción de metano del $70 \%$ en relación al SM.

Se identificó que las mejores condiciones de operación se desarrollaron en el SB con $\mathrm{pH}(6,38-8,0)$, FOSTAC $(0,42-0,60)$, AGV (160-423mg/l), remoción DQO (61$85 \%)$.

\section{LITERATURA CITADA}

APHA, AWWA, WEF. 2012. Standard Methods for examination of water and wastewater. (22nd ed.), Washington: American Public Health Association, 1360. doi:ISBN 978-087553-013-0.

Aristizábal , C. 2015. Caracterización físico-química de una vinaza resultante de la producción de alcohol de una industria licorera, a partir del aprovechamiento de la caña de azúcar. Ingenierías USBMed, 6(2): 3641.
Ashley, N., y Hurst, T. 1981. Acid and alkaline phosphatase activity in anaerobic digested sludge: a biochemical predictor of digester failure. Water Res, 15: 633-638.

Blinová, L., Sirotiak, M., Bartošová, A., y Soldán, M. 2017. Utilization of waste from coffee production. Research Papers Faculty of Materials Science and Technology Slovak University of Technology, 25(40): 91-101.

Bolte, J., Hill, D., y Wood, T. 1986. Anaerobic Digestion of Screened Swine Waste Liquids in Suspended Particle-Attached Growth Reactors. Transactions of the ASAE, 29(2):543-549.

Braun, R., Huber, P., y Meyrath, J. 1981. Ammonia toxicity in liquid piggery manure digestion. Biotechnology Letters, 3(4): 159-164.

Chen, Y., Cheng, J., y Creamer, K. 2008. Inhibition of anaerobic digestion process: a review. Bioresource technology, 99:4044-4064. doi:10.1016/j.biortech.2007.01.057.

Chinchilla, J. 2013. Evaluación de la producción de metano en el tratamiento de los residuos del café mediante la digestión anaerobia en dos fases. (Tesis pregrado). Escuela Agrícola Panamericana, Zamorano, Honduras. Recuperado el 9 de junio de 2020, de http://hdl.handle.net/11036/1764.

Crites, R., y Tchobanoglous, G. 2000. Tratamiento de aguas residuales en pequeñas poblaciones. McGrawHill. doi:ISBN: 9789584100429.

Diaz, J. 2018. Ecuaciones y cálculos para el tratamiento de aguas. Madrid, España: Ediciones Paraninfo, SA. doi:ISBN: 978-84-283-4152-3.

Dinsdale, R., Hawkes, F., y Hawkes, D. 1997. Mesophilic and thermophilic anaerobic digestion with thermophilic pre-acidification of instant-coffeeproduction wastewater. Water research, 31(8): 19311938. doi:10.1016/S0043-1354(97)00041-9.

El Telégrafo. 2017. Cafeteros de Ecuador se reunieron con empresarios rusos para fortalecer exportaciones. Recuperado el 25 de mayo de 2019, de https://www.eltelegrafo.com.ec/noticias/economia/1/ cafeteros-de-ecuador-se-reunieron-con-empresariosrusos-para-fortalecer-exportaciones.

Failla, S., y Restuccia, A. 2014. Methane potentials from grape marc by a laboratory scale plant. Applied Mathematical Sciences, 8(132): 6665-6678. 
FAO (Food and agriculture organization of the United Nations). 2013. La bioenergía y los biocombustibles. Vías de la sostenibilidad. Recuperado el 30 de mayo de 2019, de http://www.fao.org/3/a-ar589s.pdf.

Fernandez, N., y Forster, C. 1993. A study of the operation of mesophilic and thermophilic anaerobic filters treating a synthetic coffee waste. Bioresource technology, 45(3): 223-227.

Fisgativa, H., Tremier, A., Le Roux, S., Bureau, C., y Dabert, P. 2017. Understanding the anaerobic biodegradability of food waste: Relationship between the typological, biochemical and microbial characteristics. Environmental Management, 188: 95-107.

García, S., Herrera, M., Behling, E., Gutíerrez, E., Chacín, E., y Fernández, N. 1995. Inhibidor natural de las aguas residuales de procesado de café en el tratamiento anaerobio. Ciencia, 3(3).

Göblös, S., Portörő, P., Bordás, D., Kálmán, M., y Kiss, I. 2008. Comparison of the effectivities of two-phase and single-phase anaerobic sequencing batch reactors during dairy wastewater treatment. Renewable Energy, 33(5): 960-965.

González, M., Pérez, S., Wong, A., Bello, R., y Yanez, G. 2015. Residuos agroindustriales con potencial para la producción de metano mediante la digestión anaerobia. Revista Argentina de microbiología, 47(3): 229-235.

González, M. 2009. Producción de bioenergía en el norte de México: Tan lejos y tan cerca. Frontera Norte, 21(41): 177-183.

Guardia, Y., Rodríguez, S., Jiménez, J., y Sánchez, V. 2014. Performance of a UASB reactor treating coffee wet wastewater. Revista Ciencias Técnicas Agropecuarias, 23(2):50-56.

Guardia, Y. 2012. Estudio de la digestión anaerobia en dos fases para el tratamiento de las aguas residuales de despulpe del beneficiado húmedo del café. (Tesis Doctoral). Universidad Politécnica de Madrid, Madrid. Recuperado el 1 de junio de 2019, de http://oa.upm.es/14684/.

Lazcano, T. 2015. Evolución anual de la producción mundial de café: La producción mundial de café ha crecido más del $50 \%$ en los últimos 25 años.

Lossie, U., y Pütz, P. 2011. Control orientado de plantas de biogás con la ayuda de FOS/TAC. Analitica de Laboratorio valoración FOS/TAC, 1-4. Alemania.
Recuperado el 22 de junio de 2020, de https://www.interempresas.net/FeriaVirtual/Catalogo s_y_documentos/87050/FOSTAC.pdf.

Morales, C., y Vera, J. 2018. Producción de metano a partir de las aguas residuales generadas en la Industria del café instantáneo en régimen Termofílico continuo con presencia de taninos. Segunda Convencion cientifica Internacional de la UTM 2018. Universidad Tecnica de Manabí, Portoviejo. Recuperado el 10 de febrero de 2020.

Murthy, P., y Naidu, M. 2012. Sustainable management of coffee industry by-products and value additionA review. Resources, Conservation and recycling, 66: 45-58.

Mussatto, S., Machado, E., Martins, S., y Teixeira, J. 2011. Production, composition, and application of coffee and its industrial residues. Food and Bioprocess Technology, 4(5): 661-672.

Peñaranda, L., Montenegro, S., y Giraldo, P. 2017. Aprovechamiento de residuos agroindustriales en Colombia. Investigación Agraria y Ambiental, 8(2):141-150.

Quintero, M., y Rondón, Y. 2012. Estudio preliminar de la producción de biogás a partir de la Digestión anaerobia del mucílago de café utilizando lodo estiercol del cerdo como inóculo (Doctoral dissertation). (Tesis pregrado). Universidad Industrial de Santander, Bucaramanga. Recuperado el 31 de agosto de 2019, de http://tangara.uis.edu.co/biblioweb/tesis/2012/14523 4.pdf.

Ramírez, S. 2012. Aprovechamiento de residuos agroindustriales, cascarilla de arroz (oriza sativa) y residuos de papa (solanum tuberosum) para la producción de trichoderma spp. (Tesis pregrado). Universidad Técnica de Ambato, Ambato. Recuperado el 31 de agosto de 2019, de http://repositorio.uta.edu.ec/bitstream/123456789/30 63/1/SBQ.29.pdf.

Restrepo, A., Rodríguez, E., y Manjarrés, K. 2011. Cortezas de naranja comestibles: una aproximación al desarrollo de productos con valor agregado a partir de residuos agroidustriales. Produccion + Limpia, 6(2): 47-57.

Rosas, D., Ortiz, H., Herrera, J., y Leyva, O. 2016. Revalorización de algunos residuos agroindustriales y su potencia de aplicación a suelos agrícolas. Agro Productividad, 9(8):18-23. 
Saval, S. 2012. Aprovechamiento de Residuos Agroindustriales: Pasado, Presente y Futuro. BioTecnología, 16(2):14-46.

Scano, E., Asquer, C., Pistis, A., Ortu, L., Demontis, V., y Cocco, D. 2014. Biogas from anaerobic digestion of fruit and vegetable wastes: Experimental results on pilot-scale and preliminary performance evaluation of a full-scale power plant. Energy Conversion and Management, 7:22-30.

Speece, R., y Parking, G. 1983. The response of methane bacteria to toxicity. Proceedings 3rd International Symposium on Anaerobic Digestion, (págs. 23-35). Boston.

Valdez, I., y Poggi, H. 2009. Hydrogen production by fermentative consortia. Renewable and Sustainable Energy Reviews, 13(5): 1000-1013.

Vargas, Y., y Pérez, L. 2018. Aprovechamiento de residuos agroindustriales para el mejoramiento de la calidad del ambiente. Facultad de Ciencias Básicas, 14(1): 59-72.
Varnero, M. 2011. Manual de biogás. FAO. (Food and agriculture organization of the United Nations), Chile. Recuperado el 31 de mayo de 2019, de http://www.fao.org/docrep/019/as400s/as400s.pdf.

Verein Deutscher Ingenieure (VDI 4630). 2006. Fermentation of organic materials, characterisation of the substrate, sampling, collection of material data, fermentation tests. Berlin. Recuperado el 20 de febrero de 2020, de https://www.vdi.de/fileadmin/pages/vdi_de/redakteu re/richtlinien/inhaltsverzeichnisse/2385990.pdf.

Yánez, J. 2018. Estudio de la película plateada del café para su reutilización en panadería. Ecuador. (Tesis pregrado). Universidad Regional Autónoma de los Andes, Ambato. Recuperado el 23 de mayo de 2019, de

http://dspace.uniandes.edu.ec/handle/123456789/801 6. 